H. KOJIMA

KODAI MATH. J.

22 (1999), 99-115

\title{
REMARK ON FOURIER COEFFICIENTS OF MODULAR FORMS \\ OF HALF INTEGRAL WEIGHT BELONGING TO KOHNEN'S SPACES II
}

\author{
HISASHI KOJIMA
}

\section{Introduction}

In [13], Waldspurger first found that the square of Fourier coefficients $a(n)$ at a square free integer $n$ of modular forms $f(z)=\sum_{n=1}^{\infty} a(n) e[n z]$ of half integral weight is essentially proportional to the central value of the zeta function at a certain integer attached to the modular form $F$ if $f$ corresponds to $F$ by the Shimura correspondence $\Psi$ and $f$ is an eigen-function of Hecke operators. Kohnen-Zagier [1], [3] determined explicitly the constant of the proportionality in the case of modular forms belonging to Kohnen's spaces $S_{(2 k+1) / 2}(N, \chi)$ of weight $(2 k+1) / 2$ and of square free level $N$ with character $\chi$ which is a subspace of $S_{(2 k+1) / 2}\left(4 N, \chi_{1}\right)$, where $S_{(2 k+1) / 2}\left(4 N, \chi_{1}\right)$ means the space of modular cusp forms of half integral weight given in [9]. Kohnen-Zagier [1] (resp. Kohnen [3]) treated the case where $N=1$ (resp. $N$ is an odd square free integer and $\chi$ is the trivial character of level $N$ ) (cf. Kojima [4] and [5]).

In [12], Shimura intended to generalize such formulas to the case of Hilbert modular forms $f$ of half integral weight and succeeded in obtaining many general interesting formulas. Among these, some explicit and useful formulas about the proportionality constant were formulated under assumptions that $f$ satisfies the multiplicity one theorem. For modular forms belonging to Kohnen's spaces $S_{(2 k+1) / 2}(N, \chi)$, Shimura [12] did not give the same explicit formula as that of Kohnen and Zagier [1], [3].

In [6], we represented explicitly the ratio of the square of Fourier coefficients $a(n)$ at a fundamental discriminant $n$ of modular forms $f(z)=\sum_{\varepsilon(-1)^{k} n \equiv 0,1(4), n>0}$ $a(n) e[n z]$ of weight $(2 k+1) / 2$ belonging to the Kohnen's space $S_{(2 k+1) / 2}(N, \chi)$ by the central value of the zeta function of the modular form $F$ which is the image of $f$ under the Shimura correspondence $\Psi$, where $\chi$ is any primitive character modulo $N$ and $n$ is equal to $4 \tau$ with a square free integer $\tau$ satisfying $\tau \equiv$ $2,3(\bmod 4)$.

The purpose of this paper is to remove these conditions about $\chi$ and $n$ and to

Received June 10, 1998; revised October 21, 1998. 
derive a generalization of results in [6] in the case where $N$ is any odd integer, $\chi$ is an arbitrary primitive character modulo $N$ and $n$ is an arbitrary positive fundamental discriminant. Though the method of our proof is more complicated, it is the same as that of [6]. To obtain our results we need to modify slightly its method.

Section 0 is a preliminary section. In Section 1, we shall summarize some results about Kohnen's spaces, the Shimura correspondence, theta functions and Hecke operators of Kohnen's spaces. In Section 2, using these and the assumption (1-12), we show that $\left\langle\Theta\left(z, w ; \zeta_{Z}\right), g(2 w)\right\rangle=C_{\tau} L_{\tau}(f)(z)+D_{\tau} L_{\tau}(f) \mid$ $U(4)(z)$ for a modular form $f \in S_{(2 k+1) / 2}(N, \chi)$ with some constants $C_{\tau}$ and $D_{\tau}$, where $\Theta\left(z, w ; \zeta_{\boldsymbol{Z}}\right)$ is a theta function, $g(w)$ is an element in $S_{2 k}\left(N, \chi^{2}\right)$ determined by $f$ under the Shimura correspondence $\Psi, U(4)$ is the operator given by $U(4)\left(\sum_{n=1}^{\infty} b(n) e[n z]\right)=\sum_{n=1}^{\infty} b(4 n) e[n z]$ and $L_{\tau}(f)(z)=f(\tau z) \tau^{k}$. Moreover, executing an explicit and complicate computation of the image $\Psi(f)$ of $f$ under the Shimura correspondence $\Psi$, we shall verify that $C_{\tau}=\overline{a(n)} \tilde{c}_{1}$ and $D_{\tau}=\overline{a(n)} \tilde{c}_{2}$ with a fundamental discriminant $n$, where $\tilde{c}_{1}$ and $\tilde{c}_{2}$ are explicitly calculable constants. These computations are essential parts for our arguments and these formulas play an important role for our later treatments. In Section 3, using integral formulas of Section 2, the computation of the image of a product of theta series and Eisenstein series by Shimura correspondence and the method of the Rankin's convolution, under some assumptions on $f$, we shall derive an explicit connection between the square of Fourier coefficients $a(n)$ at an arbitrary positive fundamental discriminant $n$ of a modular form $f(z)=\sum_{\varepsilon(-1)^{k} n \equiv 0,1(4), n>0}$ $a(n) e[n z]$ belonging to Kohnen's spaces $S_{(2 k+1) / 2}(N, \chi)$ of half integral weight $(2 k+1) / 2$ and of an odd level $N$ with arbitrary character $\chi$ and the central value of zeta functions associated with the image $\Psi(f)$ of $f$ by the Shimura correspondence $\Psi$.

We mention that our results give a generalization of some results in KohnenZagier [1] and [3] and Kojima [4], [5] and [6] and our method can be applicable to the case of Maass wave forms of half integral weight (cf. [7]).

Finally, the author is indebted to the referee suggesting some revisions of this paper.

\section{§0. Notation and preliminaries}

We denote by $\boldsymbol{Z}, \boldsymbol{Q}, \boldsymbol{R}$ and $\boldsymbol{C}$ the ring of rational integers, the rational number field, the real number field and the complex number field, respectively. For $z \in \mathbf{C}$, we put $e[z]=\exp (2 \pi i z)$ and we define $\sqrt{z}=z^{1 / 2}$ so that $-\pi / 2<$ $\arg z^{1 / 2} \leqq \pi / 2$. Further, we set $z^{k / 2}=(\sqrt{z})^{k}$ for every $k \in Z$. Let $S L(2, \boldsymbol{R})$ denote the group of all real matrices of degree 2 with determinant one and $\mathfrak{H}$ the complex upper half plane, i.e.,

$$
S L(2, \boldsymbol{R})=\left\{\left(\begin{array}{ll}
a & b \\
c & d
\end{array}\right) \mid a, b, c \text { and } d \in \boldsymbol{R} \text { and } a d-b c=1\right\}
$$


and

$$
\mathfrak{H}=\{z=x+i y \mid x, y \in \boldsymbol{R} \text { and } y>0\} .
$$

Define an action of $S L(2, \boldsymbol{R})$ on $\mathfrak{H}$ by

$$
z \rightarrow \gamma(z)=\frac{a z+b}{c z+d} \quad \text { for all } \gamma=\left(\begin{array}{ll}
a & b \\
c & d
\end{array}\right) \in S L(2, \boldsymbol{R}) \text { and for all } z \in \mathfrak{H} \text {. }
$$

For positive integers $M$ and $M^{\prime}$, put

$$
\Gamma_{0}(M)=\left\{\left(\begin{array}{ll}
a & b \\
c & d
\end{array}\right) \in S L(2, \boldsymbol{R}) \mid a, b, c \text { and } d \in \boldsymbol{Z} \text { and } c \equiv 0(\bmod M)\right\}
$$

and

$$
\Gamma\left[M, M^{\prime}\right]=\left\{\left(\begin{array}{ll}
a & b \\
c & d
\end{array}\right) \in \Gamma_{0}\left(M^{\prime}\right) \mid b \equiv 0(\bmod M)\right\} .
$$

We introduce an automorphic factor $j_{0}(\gamma, z)$ of $\Gamma_{0}(4)$ determined by $j_{0}(\gamma, z)=\vartheta_{0}(\gamma(z)) / \vartheta_{0}(z)$ for every $\gamma=\left(\begin{array}{ll}a & b \\ c & d\end{array}\right) \in \Gamma_{0}(4)$ and for every $z \in \mathfrak{H} \quad$ with $\vartheta_{0}(z)=\sum_{n=-\infty}^{\infty} e\left[n^{2} z\right]$ and $\vartheta(z)=\vartheta_{0}(z / 2)$.

\section{§1. Shimura correspondences of modular forms of half integral weight and theta functions}

This section is devoted to summarizing several fundamental facts which we need later. Let $k$ be a positive integer. Let $N$ denote a positive integer and $\psi_{0}$ a Dirichlet character modulo $4 N$. We denote by $S_{(2 k+1) / 2}\left(4 N, \psi_{0}\right)$ the set of all cusp forms $f$ on $\mathfrak{H}$ such that

$$
f(\gamma(z))=\psi_{0}(d) j_{0}(\gamma, z)^{2 k+1} f(z) \text { for every } \gamma=\left(\begin{array}{ll}
a & b \\
c & d
\end{array}\right) \in \Gamma_{0}(4 N) .
$$

Throughout the rest of the paper we assume that $N$ is an odd integer. Let $\chi$ be a Dirichlet character modulo $N$ such that $\chi(-1)=\varepsilon$. Put $\chi_{1}=\left(\frac{4 \varepsilon}{*}\right) \chi$. We introduce a subspace $S_{(2 k+1) / 2}(N, \chi)$ of $S_{(2 k+1) / 2}\left(4 N, \chi_{1}\right)$ defined by

$$
S_{(2 k+1) / 2}(N, \chi)=\left\{f(z) \in S_{(2 k+1) / 2}\left(4 N, \chi_{1}\right) \mid f(z)=\sum_{\varepsilon(-1)^{k} n \equiv 0,1(4), n>0} a(n) e[n z]\right\} .
$$

We call $S_{(2 k+1) / 2}(N, \chi)$ the Kohnen's space of weight $(2 k+1) / 2$ and of level $N$ with character $\chi$. We denote by $S_{2 k}(M, \omega)$ the space of all cusp forms $f^{\prime}$ of 
weight $2 k$ and of level $M$ with character $\omega$ satisfying

$$
f^{\prime}(\gamma(z))=\omega(d)(c z+d)^{2 k} f^{\prime}(z) \text { for every } \gamma=\left(\begin{array}{ll}
a & b \\
c & d
\end{array}\right) \in \Gamma_{0}(M) .
$$

Here we recall the notation and results in Shimura [12]. Let $\mathfrak{b}$ and $\mathfrak{b}^{\prime}$ denote integral ideals of $\boldsymbol{Q}$ and $\psi$ a Hecke character of $\boldsymbol{Q}$ whose conductor divides $4 \mathfrak{b b}^{\prime}$. Let $\mathscr{M}_{(2 k+1) / 2}\left(\mathfrak{b}, \mathfrak{b}^{\prime} ; \psi\right)$ (resp. $\mathscr{S}_{(2 k+1) / 2}\left(\mathfrak{b}, \mathfrak{b}^{\prime} ; \psi\right)$ ) be the space of modular forms (resp. modular cusp forms) of half integral weight $(2 k+1) / 2$ given in [12, p. 507]. Let $\psi_{0}$ be a Dirichlet character modulo $4 N$ such that $\psi_{0}(-1)=1$. We choose the Hecke character $\psi$ of $\boldsymbol{Q}$ such that

$$
\prod_{p \mid 4 N} \psi_{p}(a)=\left(\frac{-1}{a}\right)^{k} \psi_{0}(a)^{-1} \quad \text { for every } a \in(Z / 4 N Z)^{\times}, \psi_{p}\left(Z_{p}^{\times}\right)=1
$$

for every $p \nmid 4 N$ and $\psi_{a}(x)=(\operatorname{sgn}(x))^{k}(x \in \boldsymbol{R})$.

For $f^{\prime} \in \mathscr{S}_{(2 k+1) / 2}(\boldsymbol{Z}, N \boldsymbol{Z} ; \psi)$, put $L\left(f^{\prime}\right)(z)=f^{\prime}(2 z)$. Then the following mapping is bijective (cf. [12, p. 523]).

$$
L: \mathscr{S}_{(2 k+1) / 2}(Z, N Z ; \psi) \rightarrow S_{(2 k+1) / 2}\left(4 N, \psi_{0}\right) .
$$

Let $t$ denote a positive square free integer. Define a mapping $\Psi_{(2 k+1) / 2, t}^{4 N, \psi_{0}}$ of $S_{(2 k+1) / 2}\left(4 N, \psi_{0}\right)$ into $S_{2 k}\left(2 N, \psi_{0}^{2}\right)$ by

$$
\Psi_{(2 k+1) / 2, t}^{4 N, \psi_{0}}(f)(z)=\sum_{m=1}^{\infty}\left(\sum_{d \mid m} \psi_{0}(d)\left(\frac{-1}{d}\right)^{k}\left(\frac{t}{d}\right) d^{k-1} a\left(t(m / d)^{2}\right)\right) e[m z]
$$

for every $f(z)=\sum_{n=1}^{\infty} a(n) e[n z] \in S_{(2 k+1) / 2}\left(4 N, \psi_{0}\right)$. The existence of this mapping was first shown by Shimura [9] and it was reformulated by Niwa [8].

Next we recall the definition of theta functions given in [12]. Put

$$
V=\left\{\xi=\left(\begin{array}{ll}
a & b \\
c & d
\end{array}\right) \in M_{2}(\boldsymbol{Q}) \mid \operatorname{tr} \xi=0\right\} \text { and }
$$

$\mathscr{S}(V)=\{\eta: V \rightarrow C \mid \eta$ is a locally constant function in the sense of [12] $\}$. by

Let $\eta$ be an element of $\mathscr{S}(V)$. Define a theta function $\Theta(z, w ; \eta)$ on $\mathfrak{H} \times \mathfrak{H}$

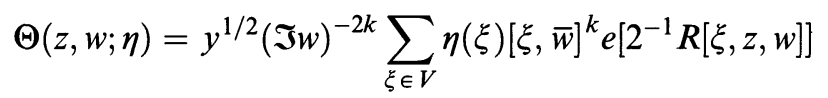

for every $(z, w) \in \mathfrak{H} \times \mathfrak{H}$, where

$$
\begin{aligned}
{[\xi, w] } & =[\xi, w, w],\left[\xi, w, w^{\prime}\right]=\left(c w w^{\prime}+d w-a w^{\prime}-b\right) \quad \text { and } \\
R[\xi, z, w] & =(\operatorname{det} \xi) z+\frac{i y}{2} \mathfrak{I} w^{-2}|[\xi, w]|^{2}\left(\xi=\left(\begin{array}{ll}
a & b \\
c & d
\end{array}\right) \in V, y=\mathfrak{I} z\right) .
\end{aligned}
$$


Throughout the rest of the paper, we take a Dirichlet character $\psi_{0}$ with $\psi_{0}(-1)=$ 1 and a positive square free integer $\tau$ such that

$$
(\tau, N)=1 \text {. }
$$

Put $\varphi=\psi \varepsilon_{\tau}$ with the Hecke character $\varepsilon_{\tau}$ associated with the quadratic field $\boldsymbol{Q}(\sqrt{\tau})$. For positive rational numbers $\alpha$ and $\beta$, put

$$
\mathfrak{o}[\alpha, \beta]=\left\{x=\left(\begin{array}{ll}
a_{x} & b_{x} \\
c_{x} & d_{x}
\end{array}\right) \in M_{2}(\boldsymbol{Q}) \mid a_{x} \in \boldsymbol{Z}, b_{x} \in \alpha \boldsymbol{Z}, c_{x} \in \beta \boldsymbol{Z} \text { and } d_{x} \in \boldsymbol{Z}\right\} .
$$

Moreover, we put $e=2 N$. We consider an element $\eta \in \mathscr{S}(V)$ determined by

$$
\eta(x)= \begin{cases}0 & \text { if } x \notin \mathfrak{v}\left[e^{-1}, e\right] \\ \sum_{t \in(1 / 2 \tau) Z / 2 N Z} \varphi_{a}(t) \varphi^{*}((2 t \tau)) e\left[-b_{x} t\right] & \text { otherwise, }\end{cases}
$$

where $\varphi^{*}$ is the ideal character associated with $\varphi$ (cf. [12, p. 505]). Introduce a mapping $L_{\tau}$ of $\mathscr{S}_{(2 k+1) / 2}(\boldsymbol{Z}, N \boldsymbol{Z} ; \psi)$ into $\mathscr{S}_{(2 k+1) / 2}\left(\boldsymbol{Z}, N \tau \boldsymbol{Z} ; \psi \varepsilon_{\tau}\right)$ defined by

$$
L_{\tau}(f)(z)=f(\tau z) \tau^{k} \quad \text { for every } f(z) \in \mathscr{S}_{(2 k+1) / 2}(\boldsymbol{Z}, N \boldsymbol{Z} ; \psi) .
$$

We put $h(z)=L_{\tau}(f)(z)$. The following lemma is proved by Shimura [12, Propositions 1.4 and 1.5].

LEMMA 1.1. The notation being as above, the mapping $L_{\tau}$ gives a bijection of $\mathscr{S}_{(2 k+1) / 2}(Z, N Z ; \psi)$ onto the set

$$
\left\{h(z)=\sum_{n=1}^{\infty} a^{\prime}(n) e[n z / 2] \in \mathscr{S}_{(2 k+1) / 2}\left(\boldsymbol{Z}, N \tau \boldsymbol{Z}, \psi \varepsilon_{\tau}\right) \mid a^{\prime}(n)=0 \text { if } \tau \nmid n\right\} .
$$

Moreover,

$$
\langle f, f\rangle=\tau^{-(2 k+1) / 2} \tau\langle h, h\rangle,
$$

where for given two cusp forms $f, g$ of weight $l$ with respect to $\Gamma$, their inner product $\langle f, g\rangle$ means

$$
\langle f, g\rangle=\operatorname{vol}(\Gamma \backslash \mathfrak{S})^{-1} \int_{\Gamma \backslash \mathfrak{S}} \overline{f(z)} g(z) \mathfrak{I} z^{l} d_{\mathfrak{H}} z \text { with } d_{\mathfrak{H}} z=\frac{d x d y}{y^{2}}(x=\mathfrak{R} z, y=\mathfrak{I} z) .
$$

The following proposition is verified by Shimura [10] and [12] (cf. Niwa [8]).

Proposition 1.1. For $f(z)=\sum_{n=1}^{\infty} a(n) e[n z / 2] \in \mathscr{S}_{(2 k+1) / 2}(Z, N Z ; \psi)$, put $h(z)=L_{\tau}(f)(z)$. Suppose that $\psi_{0}, \tau$ and $\psi$ satisfy the condition (1-7) and $\eta$ is the function on $V$ determined by (1-8). Then there exists the element

$$
g_{\tau}(w)=\sum_{m=1}^{\infty}\left(\sum_{d \mid m} \psi_{0}(d)\left(\frac{-1}{d}\right)^{k}\left(\frac{\tau}{d}\right) d^{k-1} a\left(\tau(m / d)^{2}\right)\right) e[m w]
$$


belonging to $S_{2 k}\left(2 N, \psi_{0}^{2}\right)$ such that

$$
c^{\prime} g_{\tau}(w)=\int_{\Gamma[2,2 \tau N] \backslash \mathfrak{S}} h(z) \Theta(z, w ; \eta) y^{(2 k+1) / 2} d_{\mathfrak{S} z} \text { with } c^{\prime}=2^{2+k} i^{k} 4 \tau N .
$$

Next we describe basic results of Hecke operators of Kohnen's space. Let $\chi$ denote a Dirichlet character modulo $N$. We denote by $T_{(2 k+1) / 2, \chi_{1}}^{4 N}\left(p^{2}\right)$ the Hecke operator on $S_{(2 k+1) / 2}\left(4 N, \chi_{1}\right)$ given in [9]. For a positive integer $m$, we define a function $f \mid U(m)$ on $\mathfrak{H}$ by

$$
f \mid U(m)(z)=\sum_{n=0}^{\infty} a(m n) e[n z]
$$

for every function $f(z)=\sum_{n=0}^{\infty} a(n) e[n z]$ on $\mathfrak{H}$. We denote by $T_{(2 k+1) / 2, N, \chi}(p)$ $(p \nmid N)$ the Hecke operator on $S_{(2 k+1) / 2}(N, \chi)$ defined in [2, p. 42]. The following lemma was confirmed by Kohnen [2] (see also Remark in [2, p. 46]).

LEMMA 1.2. The notation being as above, for $f(z)=\sum_{n \geqq 1, \varepsilon(-1)^{k} n \equiv 0,1(4)}$ $a(n) e[n z] \in S_{(2 k+1) / 2}(N, \chi)$, Fourier expansions of $f \mid T_{(2 k+1) / 2, N, \chi}(p)(z)(p \nmid N)$ and $f \mid U\left(p^{2}\right)(z)(p \mid N)$ are given as follows:

$$
\begin{aligned}
f \mid T_{(2 k+1) / 2, N, \chi}(p)(z)= & \sum_{n \geqq 1 ; \varepsilon(-1)^{k} n \equiv 0,1(4)}\left(a\left(p^{2} n\right)+\chi(p)\left(\frac{\varepsilon(-1)^{k} n}{p}\right) p^{k-1} a(n)\right. \\
& \left.+\chi^{2}(p) p^{2 k-1} a\left(n / p^{2}\right)\right) e[n z] \quad(p \nmid N)
\end{aligned}
$$

and

$$
f \mid U\left(p^{2}\right)(z)=\sum_{n \geqq 1, \varepsilon(-1)^{k} n \equiv 0,1(4)} a\left(p^{2} n\right) e[n z] \quad(p \mid N),
$$

where $a\left(n / p^{2}\right)$ means 0 if $p^{2} \nmid n$.

Observe that $T_{(2 k+1) / 2, N, \chi}(p)$ coincides with the restriction of $T_{(2 k+1) / 2, \chi_{1}}^{4 N}\left(p^{2}\right)$ to $S_{(2 k+1) / 2}(N, \chi)$ for every odd prime $p(p \nmid N)$. Now we impose the following assumption.

$\varepsilon=\chi(-1)$ satisfies $(-1)^{k} \varepsilon>0, \chi$ is a Dirichlet character modulo $N$, $f \in S_{(2 k+1) / 2}(N, \chi)$ is an eigenfunction of all Hecke operators

$$
T_{(2 k+1) / 2, N, \chi}(p)(p \nmid N) \text { and } U\left(p^{2}\right)(p \mid N) \text {,i.e., }
$$

$$
f \mid T_{(2 k+1) / 2, N, \chi}(p)(z)=\omega(p) f(z)(p \nmid N) \text { and } f \mid U\left(p^{2}\right)(z)=\omega(p) f(z)(p \mid N)
$$

and $a\left(4 \tau_{0}\right) \neq 0$ for some square free positive integer $\tau_{0}$ such that $\tau_{0} \equiv 2,3(\bmod 4)$. 
Since $S_{(2 k+1) / 2}(N, \chi)$ is contained in $S_{(2 k+1) / 2}\left(4 N,\left(\frac{4 \varepsilon}{*}\right) \chi\right)$, we have the following diagram.

$$
\begin{aligned}
& S_{(2 k+1) / 2}(N, \chi) \subset S_{(2 k+1) / 2}\left(4 N, \psi_{0}\right) \stackrel{L^{-1}}{\longrightarrow} \mathscr{S}_{(2 k+1) / 2}(Z, N Z ; \psi) \\
& \text { with } \psi_{0}=\left(\frac{4 \varepsilon}{*}\right) \chi .
\end{aligned}
$$

By this relation, we may identify elements $f(z)$ of $S_{(2 k+1) / 2}(N, \chi)$ with those of $\mathscr{S}_{(2 k+1) / 2}(\boldsymbol{Z}, N \boldsymbol{Z} ; \psi)$. In [6], we verified the following lemma.

LEMMA 1.3. Let $f(z)=\sum_{n \geqq 1, \varepsilon(-1)^{k} n \equiv 0,1(4)} a(n) e[n z]$ be an element of $S_{(2 k+1) / 2}(N, \chi)$ satisfying the condition (1-10) and let $\tau$ be an integer satisfying the condition that $(1-7)$ and $\tau \equiv 2,3(\bmod 4)$. Then

$$
\Psi_{(2 k+1) / 2, \tau}^{4 N, \chi\left(\frac{4 \xi_{k}}{2}\right)}(f)(w)=\sum_{m=1}^{\infty}\left(\sum_{d \mid m}\left(\frac{4 \tau}{d}\right) \chi(d) d^{k-1} a\left(\tau(m / d)^{2}\right)\right) e[m w]
$$

coincides with an element $a(4 \tau) g(2 w)$ of $S_{2 k}\left(2 N, \chi^{2}\right)$, where $g(w)=\sum_{n=1}^{\infty} c(n) e[n w]$ belongs to $S_{2 k}\left(N, \chi^{2}\right)$ and

$$
\sum_{n=1}^{\infty} c(n) n^{-s}=\prod_{p}\left(1-\omega(p) p^{-s}+\chi^{2}(p) p^{2 k-1-2 s}\right)^{-1} .
$$

It is well known that $f \mid U(4)(z)$ belongs to $S_{(2 k+1) / 2}\left(4 N, \chi\left(\frac{4 \varepsilon}{*}\right)\right)$ for every $f(z) \in S_{(2 k+1) / 2}\left(4 N, \chi\left(\frac{4 \varepsilon}{*}\right)\right)$. Here we impose the further condition on $f$ in $(1-10)$.

$$
\text { If } f^{\prime} \in S_{(2 k+1) / 2}\left(4 N, \chi\left(\frac{4 \varepsilon}{*}\right)\right) \text { and } f^{\prime} \mid T_{(2 k+1) / 2, \chi\left(\frac{4 *}{*}\right)}^{4 N}\left(p^{2}\right)=\omega(p) f^{\prime}
$$

for every prime $p(p \nmid 4 \tau N)$, then $f^{\prime}(z)$ equals

$$
\tilde{c} f(z)+\tilde{c}^{\prime} f \mid U(4)(z)
$$

for some constants $\tilde{c}$ and $\tilde{c}^{\prime}$ and, if $f^{\prime \prime} \in S_{(2 k+1) / 2}\left(4 M, \chi\left(\frac{4 \varepsilon}{*}\right)\right)$

$$
(M \mid N, M \neq N) \text { satisfies } f^{\prime \prime} \mid T_{(2 k+1) / 2, \chi\left(\frac{4 k}{*}\right)}^{4 M}\left(p^{2}\right)=\omega(p) f^{\prime \prime}(p \nmid 4 N \tau),
$$

then $f^{\prime \prime}(z)=0$ moreover, $4 \tau \mid \mathfrak{h},\left(\mathfrak{h}^{-1} 4 N \tau, \tau\right)=1$ and $8 \nmid \mathfrak{h}$ if $2 \nmid \tau$, where $\omega(p)$ is the eigenvalue given in Lemma 1.3 and $\mathfrak{h}$ is the conductor of $\varphi=\psi \varepsilon_{\tau}$ with $\psi$ in (1-11). Moreover, we impose the condition that

$$
g(w) \text { is a primitive form of } S_{2 k}\left(N, \chi^{2}\right) \text { and } f \mid U(4)(z)
$$

is not a constant times $f(z)$,

where $f(z)$ and $g(w)$ are the same elements given in Lemma 1.3. Furthermore, 
we consider the condition that

$$
\text { If } 2 \mid \tau, \text { then } \varphi_{2}(1+4 x)=\varphi_{2}\left(1+4 x^{2}\right) \text { for every } x \in Z_{2},
$$

where $\varphi_{2}$ is the restriction of $\varphi$ to $\boldsymbol{Q}_{2}^{\times}$and $\boldsymbol{Z}_{2}$ (resp. $\boldsymbol{Q}_{2}$ ) means the ring of all 2adic integers (numbers).

\section{§2. Some formulas of theta integrals}

This section is devoted to confirming a key proposition concerning theta integrals. For a positive integer $a$, we define elements $\zeta^{a Z}$ and $\zeta_{a Z}$ of $\mathscr{S}(V)$ by

$$
\zeta^{a Z}(x)= \begin{cases}\bar{\varphi}_{a}\left(b_{x}\right) \bar{\varphi}^{*}\left(\left(b_{x} a^{-1} e\right)\right) & \text { if } x \in \mathfrak{o}\left[a e^{-1}, e\right] \\ 0 & \text { otherwise }\end{cases}
$$

and

$$
\zeta_{a Z}(x)= \begin{cases}\bar{\varphi}_{a}\left(b_{x}\right) \bar{\varphi}^{*}\left(\left(b_{x} a^{-1} e\right)\right) & \text { if } x \in \mathfrak{o}\left[a e^{-1}, e\right] \text { and }\left(b_{x} a^{-1} e, 4 N \tau\right)=1, \\ 0 & \text { otherwise. }\end{cases}
$$

By the same method as that of Shimura [12, Lemma 5.7], we may derive the following lemma (cf. [11]).

LEMMA 2.1. Suppose that $N$ is odd, $\tau$ is a positive square free integer and the conditions (1-7), (1-10), (1-12), (1-13) and (1-14) are satisfied. Let a be a positive integer such that $a \mathfrak{h} \mid 4 N \tau$ and $\operatorname{ord}_{p}(a \mathfrak{h})=\operatorname{ord}_{p}(4 N \tau)$ for every $p \mid \tau$. Then there are constants $M$ and $M^{\prime}$ such that

$$
\left\langle\Theta\left(z, w ; \zeta^{a Z}\right), g(2 w)\right\rangle=M h(z)+M^{\prime} h \mid U(4)(z) \quad \text { with } h(z)=L_{\tau}(f)(z),
$$

where $g(w)$ is the same function given in Lemma 1.3, $\mathfrak{h}$ is the conductor of $\varphi\left(=\psi \varepsilon_{\tau}\right)$ and $\operatorname{ord}_{p}(d)$ is the p-adic order of an integer $d$. Moreover, the above integral is equal to 0 if $4 \tau \mid$ l.c.m. $(a \mathfrak{h}, 4)$ and l.c.m. $(a \mathfrak{h}, 4) \neq 4 N \tau$.

By virtue of Proposition 1.1, Lemma 1.3 and Lemma 2.1, we may conclude the following proposition.

Proposition 2.1. Suppose that the assumption in Lemma 2.1 is satisfied. Then we have

$$
A_{\tau} h_{\tau}(z)+B_{\tau} h_{\tau} \mid U(4)(z)=\langle\Theta(z, w ; \eta), g(2 w)\rangle
$$

and

$$
C_{\tau} h_{\tau}(z)+D_{\tau} h_{\tau} \mid U(4)(z)=\left\langle\Theta\left(z, w ; \zeta_{Z}\right), g(2 w)\right\rangle
$$


with

$$
\begin{aligned}
& A_{\tau}=\overline{a(4 \tau)} \overline{c^{\prime}} \frac{\tau^{-(2 k+1) / 2+1}}{\operatorname{vol}(\Gamma[2,2 N \tau] \backslash \mathfrak{H})} \\
& \times \frac{\langle g(2 w), g(2 w)\rangle\left\langle f^{\prime}, f^{\prime}\right\rangle-\langle g(w), g(2 w)\rangle\left\langle f, f^{\prime}\right\rangle}{\langle f, f\rangle\left\langle f^{\prime}, f^{\prime}\right\rangle-\left\langle f, f^{\prime}\right\rangle\left\langle f^{\prime}, f\right\rangle} \text { if } \tau \equiv 2,3(4), \\
& B_{\tau}=\overline{a(4 \tau)} \overline{c^{\prime}} \frac{\tau^{-(2 k+1) / 2+1}}{\operatorname{vol}(\Gamma[2,2 N \tau] \backslash \mathfrak{S})} \\
& \times \frac{\langle g(w), g(2 w)\rangle\langle f, f\rangle-\langle g(2 w), g(2 w)\rangle\left\langle f^{\prime}, f\right\rangle}{\langle f, f\rangle\left\langle f^{\prime}, f^{\prime}\right\rangle-\left\langle f, f^{\prime}\right\rangle\left\langle f^{\prime}, f\right\rangle} \text { if } \tau \equiv 2,3(4), \\
& C_{\tau}=\mu\left(\mathfrak{h}^{-1} 4 N \tau\right) \varphi^{*}\left(\mathfrak{h}^{-1} 4 N \tau\right)\left(\varphi_{a}(-1) \overline{\gamma(\varphi)} \mathfrak{h}^{-1} 4 N \tau\right)^{-1} A_{\tau} \text { if } \tau \equiv 2,3(4) \text {, } \\
& D_{\tau}=\mu\left(\mathfrak{h}^{-1} 4 N \tau\right) \varphi^{*}\left(\mathfrak{h}^{-1} 4 N \tau\right)\left(\varphi_{a}(-1) \overline{\gamma(\varphi)} \mathfrak{h}^{-1} 4 N \tau\right)^{-1} B_{\tau} \text { if } \tau \equiv 2,3(4) \text {, } \\
& A_{\tau}=\overline{a(\tau)} F_{\tau}, B_{\tau}=\overline{a(\tau)} G_{\tau} \quad \text { if } \tau \equiv 1(4) \\
& F_{\tau}=\overline{c^{\prime}} \frac{\operatorname{vol}(\Gamma[2,2 \tau N] \backslash \mathfrak{H})^{-1}}{\langle f, f\rangle\left\langle f^{\prime}, f^{\prime}\right\rangle-\left\langle f, f^{\prime}\right\rangle\left\langle f^{\prime}, f\right\rangle} \tau^{-(2 k+1) / 2+1} \\
& \times\left(\left\langle f^{\prime}, f^{\prime}\right\rangle\left(\langle g(w), g(2 w)\rangle-\left(\frac{\tau}{2}\right) \overline{\chi(2)} 2^{k-1}\langle g(2 w), g(2 w)\rangle\right)\right. \\
& -\left\langle f, f^{\prime}\right\rangle\left(\left(\overline{\omega(2)}-2^{k-1} \overline{\chi(2)}\left(\frac{\tau}{2}\right)\right)\right. \\
& \left.\left.\times\langle g(w), g(2 w)\rangle-\overline{\chi\left(2^{2}\right)} 2^{2 k-1}\langle g(2 w), g(2 w)\rangle\right)\right) \\
& G_{\tau}=\overline{c^{\prime}} \frac{\operatorname{vol}(\Gamma[2,2 \tau N] \backslash \mathfrak{H})^{-1}}{\langle f, f\rangle\left\langle f^{\prime}, f^{\prime}\right\rangle-\left\langle f, f^{\prime}\right\rangle\left\langle f^{\prime}, f\right\rangle} \tau^{-(2 k+1) / 2+1} \\
& \times\left(-\left\langle f^{\prime}, f\right\rangle\left(\langle g(w), g(2 w)\rangle-\left(\frac{\tau}{2}\right) \overline{\chi(2)} 2^{k-1}\langle g(2 w), g(2 w)\rangle\right)\right. \\
& +\langle f, f\rangle\left(\left(\overline{\omega(2)}-2^{k-1} \overline{\chi(2)}\left(\frac{\tau}{2}\right)\right)\right. \\
& \left.\left.\times\langle g(w), g(2 w)\rangle-\overline{\chi\left(2^{2}\right)} 2^{2 k-1}\langle g(2 w), g(2 w)\rangle\right)\right) \\
& C_{\tau}=\mu\left(\mathfrak{h}^{-1} 4 N \tau\right) \varphi^{*}\left(\mathfrak{h}^{-1} 4 N \tau\right)\left(\varphi_{a}(-1) \overline{\gamma(\varphi)} \mathfrak{h}^{-1} 4 N \tau\right)^{-1} A_{\tau} \text { if } \tau \equiv 1(4), \\
& D_{\tau}=\mu\left(\mathfrak{h}^{-1} 4 N \tau\right) \varphi^{*}\left(\mathfrak{h}^{-1} 4 N \tau\right)\left(\varphi_{a}(-1) \overline{\gamma(\varphi)}^{-1} 4 N \tau\right)^{-1} B_{\tau} \text { if } \tau \equiv 1(4),
\end{aligned}
$$


where $\eta$ is the same element given in (1-8), $h_{\tau}(z)=L_{\tau}(f)(z), h_{\tau}^{\prime}(z)=h_{\tau} \mid U(4)(z)$, $f^{\prime}=f \mid U(4)$ and $\gamma(\varphi)$ means the Gauss sum of $\varphi$.

Proof. If $\tau \equiv 2,3(\bmod 4)$, we can verify in a manner similar to that of [6]. So we may assume that $\tau \equiv 1(\bmod 4)$. We see that

$$
\begin{aligned}
& \Psi_{(2 k+1) / 2, \tau}^{4 N, \chi\left(\frac{4 \varepsilon}{*}\right)}(f)(w) \\
& =\sum_{m=1}^{\infty}\left(\sum_{d \mid m} \chi(d)\left(\frac{4}{d}\right)\left(\frac{\tau}{d}\right) d^{k-1} a\left(\tau(m / d)^{2}\right)\right) e[m w] \in S_{2 k}\left(2 N, \chi^{2}\right) \\
& T_{2 k, \chi^{2}}^{2 N}(p)\left(\Psi_{(2 k+1) / 2, \tau}^{4 N, \chi\left(\frac{4 \varepsilon}{*}\right)}(f)\right)=\omega(p) \Psi_{(2 k+1) / 2, \tau}^{4 N, \chi\left(\frac{4 \varepsilon}{*}\right)}(f) \quad \text { and } \\
& T_{2 k, \chi^{2}}^{2 N}(p) g=\omega(p) g \text { for every prime } p((2 N, p)=1),
\end{aligned}
$$

where $T_{2 k, \chi^{2}}^{2 N}(p)$ is Hecke operators on $S_{2 k}\left(2 N, \chi^{2}\right)$. Since $g$ is a primitive form of $S_{2 k}\left(N, \chi^{2}\right)$, there are constants $c_{1}$ and $c_{2}$ such that

$$
\Psi_{(2 k+1) / 2, \tau}^{4 N, \chi\left(\frac{4 \varepsilon}{*}\right)}(f)(w)=c_{1} g(w)+c_{2} g(2 w)
$$

Therefore we obtain

$$
L\left(s-k+1, \chi\left(\frac{4}{*}\right)\left(\frac{\tau}{*}\right)\right) \sum_{n=1}^{\infty} a\left(\tau n^{2}\right) n^{-s}=\left(c_{1}+c_{2} 2^{-s}\right) L(s, g)
$$

with $L(s, g)=\sum_{n=1}^{\infty} c(n) n^{-s}$, where $L\left(s, \chi\left(\frac{4}{*}\right)\left(\frac{\tau}{*}\right)\right)$ is the Dirichlet $L$-function associated with $\chi\left(\frac{4}{*}\right)\left(\frac{\tau}{*}\right)$. By Lemma 1.2 and the condition (1-10), we have

$$
\begin{aligned}
& L\left(s-k+1, \chi\left(\frac{\tau}{*}\right)\right) \sum_{n=1}^{\infty} a\left(\tau n^{2}\right) n^{-s} \\
& =a(\tau) \prod_{p}\left(1-\omega(p) p^{-s}+\chi\left(p^{2}\right) p^{2 k-1-2 s}\right)^{-1}
\end{aligned}
$$

(cf. [7, p. 452 and p. 453]). Comparing 2-factors of Dirichlet series (2-4) and $(2-5)$, we can check

$$
\begin{aligned}
& \left(c_{1}+c_{2} 2^{-s}\right)\left(1-\omega(2) 2^{-s}+\chi\left(2^{2}\right) 2^{2 k-1-2 s}\right)^{-1} \\
& \quad=a(\tau)\left(1-\chi(2)\left(\frac{\tau}{2}\right) 2^{-s+k-1}\right)\left(1-\omega(2) 2^{-s}+\chi\left(2^{2}\right) 2^{2 k-1-2 s}\right)^{-1}
\end{aligned}
$$

which yields that

$$
c_{1}=a(\tau) \quad \text { and } \quad c_{2}=-\left(\frac{\tau}{2}\right) \chi(2) 2^{k-1} a(\tau)
$$


Therefore we may justify

$$
\Psi_{(2 k+1) / 2, \tau}^{4 N, \chi\left(\frac{4 \varepsilon}{*}\right)}(f)(w)=a(\tau) g(w)-\left(\frac{\tau}{2}\right) \chi(2) 2^{k-1} a(\tau) g(2 w) .
$$

Put $\tilde{f}(z)=f \mid U(4)(z)$. Then we see

$$
\Psi_{(2 k+1) / 2, \tau}^{4 N, \chi\left(\frac{4 k}{*}\right)}(\tilde{f})(w)=\sum_{m=1}^{\infty}\left(\sum_{d \mid m} \chi(d)\left(\frac{4}{d}\right)\left(\frac{\tau}{d}\right) d^{k-1} a\left(4 \tau(m / d)^{2}\right)\right) e[m w] .
$$

So we can put

(2-9) $\quad \Psi_{(2 k+1) / 2, \tau}^{4 N, \chi\left(\frac{4 \varepsilon}{*}\right)}(\tilde{f})(w)=c_{1}^{\prime} g(w)+c_{2}^{\prime} g(2 w)$ for some constants $c_{1}^{\prime}$ and $c_{2}^{\prime}$.

Next we shall determine explicitly $c_{1}^{\prime}$ and $c_{2}^{\prime}$. For this purpose we need to compute the Dirichlet series

$$
L\left(s-k+1, \chi\left(\frac{4}{*}\right)\left(\frac{\tau}{*}\right)\right) \sum_{n=1}^{\infty} a\left(4 \tau n^{2}\right) n^{-s} .
$$

Consider a power series $H_{n}(x)$ of $x$ defined by

$$
H_{n}(x)=\sum_{m=0}^{\infty} a\left(\tau 2^{2(m+1)} n^{2}\right) x^{m} .
$$

By the definition of $H_{n}(x)$, we obtain

$$
\begin{aligned}
x H_{n}(x)= & \sum_{m=0}^{\infty} a\left(\tau 2^{2 m} n^{2}\right) x^{m}-a\left(\tau n^{2}\right) \\
= & a\left(\tau n^{2}\right)\left(1-2^{k-1} \chi(2)\left(\frac{\tau}{2}\right) x\right) \\
& \times\left(1-\omega(2) x+\chi\left(2^{2}\right) 2^{2 k-1} x^{2}\right)^{-1}-a\left(\tau n^{2}\right)
\end{aligned}
$$

for every $n(2 \nmid n)$, which shows that

$$
H_{n}(x)=a\left(\tau n^{2}\right) \frac{\omega(2)-2^{k-1} \chi(2)\left(\frac{\tau}{2}\right)-\chi\left(2^{2}\right) 2^{2 k-1} x}{1-\omega(2) x+\chi\left(2^{2}\right) 2^{2 k-1} x^{2}} \text { for every } n(2 \nmid n) .
$$

Since

we find

$$
\sum_{n=1}^{\infty} a\left(4 \tau n^{2}\right) n^{-s}=\sum_{(n, 2)=1} H_{n}\left(2^{-s}\right) n^{-s}
$$

$$
c_{1}^{\prime}=a(\tau)\left(\omega(2)-2^{k-1} \chi(2)\left(\frac{\tau}{2}\right)\right) \quad \text { and } \quad c_{2}^{\prime}=a(\tau)\left(-\chi\left(2^{2}\right) 2^{2 k-1}\right) .
$$


Therefore we conclude that

$$
\Psi_{(2 k+1) / 2, \tau}^{4 N, \chi\left(\frac{4 \varepsilon}{)}\right)}(\tilde{f})(w)=a(\tau)\left(\left(\omega(2)-2^{k-1} \chi(2)\left(\frac{\tau}{2}\right)\right) g(w)-\chi\left(2^{2}\right) 2^{2 k-1} g(2 w)\right) .
$$

For a positive integer $a$, we put

$$
I(a)=\left\langle\Theta\left(z, w ; \zeta^{a Z}\right), g(2 w)\right\rangle .
$$

By [12, p. 539], (1-12) and Lemma 2.1, we have

$$
\begin{aligned}
\left\langle\Theta\left(z, w ; \zeta_{\boldsymbol{Z}}\right), g(2 w)\right\rangle & =\mu\left(\mathfrak{h}^{-1} 4 N \tau\right) \varphi^{*}\left(\mathfrak{h}^{-1} 4 N \tau\right) I\left(\mathfrak{h}^{-1} 4 N \tau\right) \\
& =C h_{\tau}(z)+D h_{\tau}^{\prime}(z)
\end{aligned}
$$

and

$$
\begin{aligned}
\langle\Theta(z, w ; \eta), g(2 w)\rangle & =\varphi_{a}(-1) \overline{\gamma(\varphi)}\left(\mathfrak{h}^{-1} 4 N \tau\right) I\left(\mathfrak{h}^{-1} 4 N \tau\right) \\
& =A h_{\tau}(z)+B h_{\tau}^{\prime}(z)
\end{aligned}
$$

for some constants $A, B, C$ and $D$. We shall determine explicitly these values. By (2-7), we may check

$$
\begin{aligned}
& (2-16)\left\langle h_{\tau}(z),\langle\Theta(z, w ; \eta), g(2 w)\rangle\right\rangle \\
& =\left(A\left\langle h_{\tau}, h_{\tau}\right\rangle+B\left\langle h_{\tau}, h_{\tau}^{\prime}\right\rangle\right) \\
& =\operatorname{vol}(\Gamma[2 \tau, 4 N] \backslash \mathfrak{H})^{-1} \operatorname{vol}(\Gamma[2,2 \tau N] \backslash \mathfrak{H})^{-1} \\
& \times \int_{\Gamma[2 \tau, 4 N] \backslash \mathfrak{H}}\left\{\overline{\int_{\Gamma[2,2 \tau N] \backslash \mathfrak{H}} h_{\tau}(z) \Theta(z, w ; \eta) \mathfrak{I} z^{(2 k+1) / 2} d_{\mathfrak{S}} z}\right\} g(2 w) \mathfrak{I} w^{2 k} d_{\mathfrak{S}} w \\
& =\operatorname{vol}(\Gamma[2,2 \tau N] \backslash \mathfrak{H})^{-1} \overline{c^{\prime} a(\tau)}(\langle g(w), g(2 w)\rangle \\
& \left.-\overline{\left(\frac{\tau}{2}\right) \chi(2) 2^{k-1}}\langle g(2 w), g(2 w)\rangle\right) .
\end{aligned}
$$

By (2-14), we may also justify that

(2-17) $A\left\langle h_{\tau}^{\prime}, h_{\tau}\right\rangle+B\left\langle h_{\tau}^{\prime}, h_{\tau}^{\prime}\right\rangle=\operatorname{vol}(\Gamma[2,2 \tau N] \backslash \mathfrak{H})^{-1} \overline{c^{\prime} a(\tau)} \overline{\left(\omega(2)-2^{k-1} \chi(2)\left(\frac{\tau}{2}\right)\right)}$

$$
\left.\langle g(w), g(2 w)\rangle-\overline{\chi\left(2^{2}\right)} 2^{2 k-1}\langle g(2 w), g(2 w)\rangle\right) .
$$

Therefore, by (2-15), (2-16) and (2-17), we conclude our assertions. 


\section{§3. Rankin's convolution of theta series, Eisenstein series and final calculation}

Put

$$
\vartheta(z)=\sum_{n=-\infty}^{\infty} e\left[n^{2} z / 2\right] \text { and } L_{M}(s, \omega)=\sum_{n=1}^{\infty} \omega^{*}(n \boldsymbol{Z}) n^{-s}
$$

for each Hecke character $\omega$ of $\boldsymbol{Q}$ and for each positive integer $M$, where $n$ runs over all positive integers such that $(n, M)=1$ and $\omega^{*}$ is the ideal character associated with $\omega$ (cf. [12, p. 505]). Let $g(w), f(z), h_{\tau}$ and $h_{\tau}^{\prime}(z)$ be the same functions in Lemma 2.1 and Proposition 2.1.

We consider integrals

$$
I_{1}=\int_{\Gamma \backslash \mathfrak{Y}} h_{\tau}(z) \overline{\vartheta(z) C(z, \bar{s}+1 / 2: k, \bar{\varphi}, \Gamma)} y^{(2 k+1) / 2} d_{\mathfrak{S} z} \quad(z=x+i y)
$$

and

$$
I_{2}=\int_{\Gamma \backslash \mathfrak{H}} h_{\tau}^{\prime}(z) \overline{\vartheta(z) C(z, \bar{s}+1 / 2: k, \bar{\varphi}, \Gamma)} y^{(2 k+1) / 2} d_{\mathfrak{H}} z \quad(z=x+i y),
$$

where $\Gamma=\Gamma[2,2 \tau N], C(z, s: k, \bar{\varphi}, \Gamma)=L_{4 N \tau}(2 s, \bar{\varphi}) E(z, s: k, \bar{\varphi}, \Gamma), C(z, s: k, \bar{\varphi}, \Gamma)$ and $E(z, s: k, \bar{\varphi}, \Gamma)$ means functions given in [12, (4-6) and (4-11)]. By the same method as that of Shimura [12, p. 542], we may check that

$$
I_{1}= \begin{cases}4 a(4 \tau) \tau^{k} \frac{\Gamma(s+k / 2)}{\left(2 \pi \tau^{2}\right)^{s+k / 2}} 2^{-(2 s+k)} L(2 s+k, g) & \text { if } \tau \equiv 2,3(4), \\ 4 a(\tau) \tau^{k} \frac{\Gamma(s+k / 2)}{\left(2 \pi \tau^{2}\right)^{s+k / 2}}\left(1-\left(\frac{\tau}{2}\right) \chi(2) 2^{k-1} 2^{-(2 s+k)}\right) L(2 s+k, g) & \text { if } \tau \equiv 1(4)\end{cases}
$$

and

$$
\begin{aligned}
I_{2}= & 4 a(4 \tau) \tau^{k} \frac{\Gamma(s+k / 2)}{\left(2 \pi \tau^{2}\right)^{s+k / 2}} L(2 s+k, g) \quad \text { if } \tau \equiv 2,3(4) \\
I_{2}= & 4 a(\tau) \tau^{k} \frac{\Gamma(s+k / 2)}{\left(2 \pi \tau^{2}\right)^{s+k / 2}} \\
& \times\left(\left(c(2)-2^{k-1} \chi(2)\left(\frac{\tau}{2}\right)\right)-\chi\left(2^{2}\right) 2^{2 k-1} 2^{-(2 s+k)}\right) L(2 s+k, g) \quad \text { if } \tau \equiv 1(4),
\end{aligned}
$$

where $L(s, g)=\sum_{n=1}^{\infty} c(n) n^{-s}$ and $g(w)=\sum_{n=1}^{\infty} c(n) e[n w]$.

Next we calculate an integral

$$
\int_{\Gamma[2 \tau, 4 N] \backslash \mathfrak{T}} g(2 w) \overline{C(w, \bar{s}+1 / 2: \bar{\varphi}) E(w, \bar{t}+1 / 2: \bar{\varphi})} \mathfrak{J} w^{2 k} d_{\mathfrak{H}} w
$$


where $C(w, s: \bar{\varphi})=C(w, s: k, \bar{\varphi}, \Gamma[2 \tau, 4 N])$ and $E(w, t: \bar{\varphi})=E(w, t: k, \bar{\varphi}, \Gamma[2 \tau, 4 N])$ are given in [12, (4-6) and (4-11)]. By a method similar to that of [12, p. 550], we find

$$
\begin{gathered}
\int_{\Gamma[2 \tau, 4 N] \backslash \mathfrak{H}} g(2 w) \overline{C(w, \bar{s}+1 / 2: \bar{\varphi}) E(w, \bar{s}+1 / 2, \bar{\varphi})} \mathfrak{I} w^{2 k} d_{\mathfrak{S} w} \\
=A(s) L_{4 N}(2 s+1, \varphi)^{-1} L(2 s+k, g) L(k, g, \bar{\varphi})
\end{gathered}
$$

where

$$
\begin{aligned}
A(s)= & 2 \tau \overline{\gamma(\bar{\varphi})} \mathfrak{h}^{-1}(4 N)^{-2 s} i^{-k} 2^{-(2 s+k)}(2 \pi)^{-k+1}\left(N / N^{\prime}\right)^{-k} \sum_{t \mid N / N^{\prime}} \\
& \mu(t) \varphi^{*}((t)) t^{k-1} c\left(N / t N^{\prime}\right)(2 \pi)^{-1 / 2} 2^{2 s+k-(1 / 2)}(2 \tau)^{-2 s} 2^{-k} \frac{\Gamma(s+(k / 2)) \Gamma(k)}{\Gamma(s+(k / 2)+(1 / 2))}
\end{aligned}
$$

where $\mathfrak{h}=4 \tau N^{\prime}\left(N^{\prime} \mid N\right)$.

Exchanging the order of integration, we have

$$
\begin{aligned}
& \int_{\Gamma[2,2 \tau N] \backslash \mathfrak{T}}\left\langle\Theta\left(z, w ; \zeta_{\boldsymbol{Z}}\right), g(2 w)\right\rangle \overline{\mathcal{\vartheta}(z) C(z, \bar{s}+1 / 2: k, \bar{\varphi}, \Gamma[2,2 \tau N])} y^{(2 k+1) / 2} d_{\mathfrak{H}} z \\
& =\int_{\Gamma[2,2 \tau N] \backslash \mathfrak{H}} \operatorname{vol}(\Gamma[2 \tau, 4 N] \backslash \mathfrak{H})^{-1}\left\{\int_{\Gamma[2 \tau, 4 N] \backslash \mathfrak{H}} \overline{\Theta\left(z, w ; \zeta_{\boldsymbol{Z}}\right)} g(2 w) \mathfrak{I} w^{2 k} d_{\mathfrak{H}} w\right\} \\
& \times \overline{\vartheta(z) C(z, \bar{s}+1 / 2: k, \bar{\varphi}, \Gamma[2,2 \tau N])} y^{(2 k+1) / 2} d_{\mathfrak{H} z} \\
& =\operatorname{vol}(\Gamma[2 \tau, 4 N] \backslash \mathfrak{H})^{-1} \int_{\Gamma[2 \tau, 4 N] \backslash \mathfrak{H}}\left\{\overline{\int_{\Gamma[2,2 \tau N] \backslash \mathfrak{H}} \vartheta(z) \Theta\left(z, w ; \zeta_{\boldsymbol{Z}}\right)}\right. \\
& \left.\times \overline{C(z, \bar{s}+1 / 2: k, \bar{\varphi}) y^{(2 k+1) / 2} d_{\mathfrak{G}} z}\right\} g(2 w) \mathfrak{I} w^{2 k} d_{\mathfrak{G} w} \\
& =\left\langle M^{\prime}(w, \bar{s}), g(2 w)\right\rangle
\end{aligned}
$$

with

$$
M^{\prime}(w, s)=\int_{\Gamma[2,2 \tau N] \backslash \mathfrak{H}} \vartheta(z) \Theta\left(z, w ; \zeta_{\boldsymbol{Z}}\right) C(z, s+1 / 2: k, \bar{\varphi}, \Gamma[2,2 \tau N]) y^{(2 k+1) / 2} d_{\mathfrak{H}} z
$$

For further computation, we need the following formula in [12, (7.9a) and (7.13)]

$$
\begin{aligned}
M^{\prime}(w, s)= & B(s) L_{4 N \tau}(2 s+1, \bar{\varphi})(-1)^{k} \sum_{q \in 2 N Z / 4 N Z} \\
& (2 N)^{2 s+1} C(w, s+(1 / 2) ; \bar{\varphi}) E(w, s+(1 / 2) ; \bar{\varphi}) \|_{2 k} \tau_{q}
\end{aligned}
$$


with

$$
B(s)=2 \frac{2^{s+(k / 2)+(1 / 2)}}{\pi^{s+(k / 2)+(1 / 2)}} \Gamma\left(s+\frac{k}{2}+\frac{1}{2}\right) \quad \text { and } \quad \tau_{q}=\left(\begin{array}{ll}
1 & 0 \\
q & 1
\end{array}\right),
$$

where $p \|_{l} \alpha(z)$ means $p(\alpha(z))(c z+d)^{-l}$ for a function $p(z)$ on $\mathfrak{H}$ and a $\alpha=$ $\left(\begin{array}{ll}a & b \\ c & d\end{array}\right) \in S L(2, \boldsymbol{R})$. Employing (3-13) and (3-18), we have

$$
\begin{aligned}
\left\langle M^{\prime}(w, \bar{s}), g(2 w)\right\rangle= & 2(-1)^{k} \overline{B(\bar{s})}(2 N)^{2 s+1} A(s) \operatorname{vol}(\Gamma[2 \tau, 4 N] \backslash \mathfrak{H})^{-1} \\
& \times L(2 s+k, g) L(k, g, \bar{\varphi}) .
\end{aligned}
$$

Combining Proposition 2.1 with (3-5), (3-7), we may derive that

$$
\begin{aligned}
& \int_{\Gamma[2,2 \tau N] \backslash \mathfrak{H}} C_{\tau} h_{\tau}(z) \overline{\vartheta(z) C(z, \bar{s}+1 / 2: k, \bar{\varphi}, \Gamma[2,2 \tau N])} y^{(2 k+1) / 2} d_{\mathfrak{H}} z \\
& \quad+\int_{\Gamma[2,2 \tau N] \backslash \mathfrak{H}} D_{\tau} h_{\tau}^{\prime}(z) \overline{\vartheta(z) C(z, \bar{s}+1 / 2: k, \bar{\varphi}, \Gamma[2,2 \tau N])} y^{(2 k+1) / 2} d_{\mathfrak{H}} z \\
&=2(-1)^{k} \overline{B(\bar{s})}(2 N)^{2 s+1} A(s) \operatorname{vol}(\Gamma[2 \tau, 4 N] \backslash \mathfrak{H})^{-1} L(2 s+k, g) L(k, g, \bar{\varphi}) .
\end{aligned}
$$

Applying the formulas (3-2) and (3-8), we multiply both sides of (3-8) by $L(2 s+k, g)^{-1}$. Moreover, by Proposition 2.1, (3.2) and (3.8), putting $2 s+k=0$, we may deduce that

$$
\begin{aligned}
|a(4 \tau)|^{2} & \frac{\overline{c^{\prime}} \mu\left(\mathfrak{h}^{-1} 4 N \tau\right) \varphi^{*}\left(\mathfrak{h}^{-1} 4 N \tau\right) E}{\varphi_{a}(-1) \overline{\gamma(\varphi)} \mathfrak{h}^{-1} 4 N \tau} \\
= & \tau^{(2 k+1) / 2-1}(4 N \tau) \mathfrak{h}^{-1} i^{k} \overline{\gamma(\bar{\varphi})} 2^{1 / 2} \pi^{-k}(k-1) ! \frac{\operatorname{vol}(\Gamma[2,2 N \tau] \backslash \mathfrak{Y})}{\operatorname{vol}(\Gamma[2 \tau, 4 N] \backslash \mathfrak{H})} \\
& \times\left(N / N^{\prime}\right)^{-k} \sum_{t \mid N / N^{\prime}} \mu(t) \varphi^{*}((t)) t^{k-1} c\left(N / t N^{\prime}\right) L(k, g, \bar{\varphi}) \quad \text { if } \tau \equiv 2,3(4)
\end{aligned}
$$

and

$$
\begin{aligned}
4|a(\tau)|^{2} & \tau^{k} \mu\left(\mathfrak{h}^{-1} 4 N \tau\right) \varphi^{*}\left(\left(\mathfrak{h}^{-1} 4 N \tau\right)\right)\left(\varphi_{a}(-1) \overline{\gamma(\varphi)} \mathfrak{h}^{-1} 4 N \tau\right)^{-1} \\
& \times\left(F_{\tau}\left(1-\left(\frac{\tau}{2}\right) \chi(2) 2^{k-1}\right)+G_{\tau}\left(\left(c(2)-2^{k-1} \chi(2)\left(\frac{\tau}{2}\right)\right)-\chi\left(2^{2}\right) 2^{2 k-1}\right)\right) \\
= & (-1)^{k} 2 \frac{2^{1 / 2}}{\pi^{1 / 2}} 2(2 N)^{-k+1} 2 \tau \overline{\gamma(\bar{\varphi})} \mathfrak{h}^{-1}(4 N)^{k} i^{-k} \\
& \times(2 \pi)^{-k+1}\left(N / N^{\prime}\right)^{-k} \sum_{t \mid N / N^{\prime}} \mu(t) \varphi^{*}((t)) t^{k-1} c\left(N / t N^{\prime}\right)(2 \pi)^{-1 / 2} \\
& \times 2^{-1 / 2}(2 \tau)^{k} 2^{-k}(k-1) ! \operatorname{vol}(\Gamma[2 \tau, 4 N] \backslash \mathfrak{H})^{-1} L(k, g, \bar{\varphi}) \text { if } \tau \equiv 1(4)
\end{aligned}
$$


with $E=\frac{\langle g(2 w), g(2 w)\rangle\left(\left\langle f^{\prime}, f^{\prime}\right\rangle-\left\langle f^{\prime}, f\right\rangle\right)+\langle g(w), g(2 w)\rangle\left(\langle f, f\rangle-\left\langle f, f^{\prime}\right\rangle\right)}{\langle f, f\rangle\left\langle f^{\prime}, f^{\prime}\right\rangle-\left\langle f, f^{\prime}\right\rangle\left\langle f^{\prime}, f\right\rangle}$, where $F_{\tau}$ (resp. $G_{\tau}$ ) is the quantity given in Proposition 2.1 and $f^{\prime}(z)=f \mid U(4)(z)$.

Consequently, by (3-9), we conclude the following theorem.

THEOREM. Let the notation be as above. Suppose that $N$ is odd, $\tau$ is a positive square free integer and the conditions (1-7), (1-10), (1-12), (1-13) and (1-14) are satisfied. Then we have

$$
\begin{aligned}
|a(4 \tau)|^{2} E= & 2^{-(1 / 2)-k} N \\
& \times \tau^{(2 k+1) / 2} \pi^{-k} \mathfrak{h}^{-2}(k-1) ! \overline{\gamma(\varphi) \gamma(\bar{\varphi})}\left(\mu\left(\mathfrak{h}^{-1} 4 N \tau\right) \varphi^{*}\left(\mathfrak{h}^{-1} 4 N \tau\right)\right)^{-1}(-1)^{k} \\
& \times \varphi_{a}(-1)\left(N / N^{\prime}\right)^{-k} \sum_{t \mid N / N^{\prime}} \mu(t) \varphi^{*}((t)) t^{k-1} c\left(N / t N^{\prime}\right) L(k, g, \bar{\varphi}) \text { if } \tau \equiv 2,3(4)
\end{aligned}
$$

and

$$
\begin{aligned}
|a(\tau)|^{2} & \left(F_{\tau}\left(1-\left(\frac{\tau}{2}\right) \chi(2) 2^{k-1}\right)\right. \\
& \left.+G_{\tau}\left(\left(c(2)-2^{k-1} \chi(2)\left(\frac{\tau}{2}\right)\right)-\chi\left(2^{2}\right) 2^{2 k-1}\right)\right) \\
= & i^{k} 2^{5 / 2} \pi^{-k} N \tau \mathfrak{h}^{-1} \overline{\gamma(\bar{\varphi})}\left(N / N^{\prime}\right)^{-k}\left(\mu\left(\mathfrak{h}^{-1} 4 N \tau\right) \varphi^{*}\left(\left(\mathfrak{h}^{-1} 4 N \tau\right)\right)\right)^{-1} \\
& \times \varphi_{a}(-1) \overline{\gamma(\varphi)} \mathfrak{h}^{-1} 4 N \tau \sum_{t \mid N / N^{\prime}} \mu(t) \varphi^{*}((t)) t^{k-1} c\left(N / t N^{\prime}\right) \\
& \times(k-1) ! \operatorname{vol}(\Gamma[2 \tau, 4 N] \backslash \mathfrak{S})^{-1} L(k, g, \bar{\varphi}) \quad \text { if } \tau \equiv 1(4)
\end{aligned}
$$

where $\mathfrak{h}$ is the conductor of $\varphi\left(=\psi \varepsilon_{\tau}\right), \mathfrak{h}=4 \tau N^{\prime}$ and $\mu(n)$ is the Möbius function of $n$.

\section{REFERENCES}

[ 1] W. Kohnen AND D. Zagier, Values of $L$-series of modular forms at the center of the critical strip, Invent. Math., 64 (1981), 175-198.

[2] W KoHnen, Newforms of half integral weight, J. Reine Angew. Math., 333 (1982), 32-72.

[3] W. KoHNEN, Fourier coefficients of modular forms of half-integral weight, Math. Ann., 271 (1985), 237-268.

[4] H. KoJima, Remark on Kohnen-Zagıer's paper concernıng Fourier coefficients of modular forms of half integral weight, Proc. Japan Acad., 69 (1993), 383-388.

[5] H. Kojima, Fourier coefficients of modular forms of half integral weight, the special values of zeta functions and Eisensteın series, preprint.

[6] H. KoJima, Remark on Fourier coefficients of modular forms of half integral weight belonging to Kohnen's spaces, to appear in J. Math. Soc. Japan. 
[7] H. Kojima, On Fourier coefficients of Maass wave forms of half integral weight belonging to Kohnen's spaces, to appear in Tsukuba J. Math.

[8] S. NiwA, Modular forms of half-integral weight and the integral of certain theta functions, Nagoya Math. J., 56 (1974), 147-161.

[9] G. SHimurA, On modular forms of half integral weight, Ann. of Math., 97 (1973), 440-481.

[10] G. SHImURA, On Hilbert modular forms of half-integral weight, Duke Math. J., 55 (1987), $765-838$.

[11] G. SHimura, On the critical values of certain Dirıchlet series and the periods of automorphic forms, Invent. Math., 94 (1988), 245-305.

[12] G. Shimura, On the Fourier coefficients of Hilbert modular forms of half-integral weight, Duke Math. J., 71 (1993), 501-557.

[13] J.-L. WALDSPURGER, Sur les coefficients de Fourier des formes modulaires de poids demi-entier, J. Math. Pures Appl., 60 (1981), 375-484.

DEPARTMENT OF MATHEMATICS

FACULTY OF EDUCATION

IWATE UNIVERSITY

MORIOKA 020

JAPAN

E-mail: kojima@iwate-u.ac.jp 